\title{
Th2-predominant inflammation and blockade of IFN- $\gamma$ signaling induce aneurysms in allografted aortas
}

\author{
Koichi Shimizu, ${ }^{1}$ Masayoshi Shichiri, ${ }^{2}$ Peter Libby, ${ }^{1}$ Richard T. Lee, ${ }^{1}$ and Richard N. Mitchell ${ }^{3}$ \\ ${ }^{1}$ The Donald W. Reynolds Cardiovascular Clinical Research Center, Cardiovascular Division, Department of Medicine, Brigham and Women's Hospital, \\ Harvard Medical School, Boston, Massachusetts, USA. ${ }^{2}$ Tokyo Medical and Dental University, Tokyo, Japan. ${ }^{3}$ Department of Pathology, \\ Brigham and Women's Hospital, Harvard Medical School, Boston, Massachusetts, USA.
}

\begin{abstract}
Abdominal aortic aneurysms (AAAs) cause death due to complications related to expansion and rupture. The underlying mechanisms that drive AAA development remain largely unknown. We recently described evidence for a shift toward T helper type 2 (Th2) cell responses in human AAAs compared with stenotic atheromas. To evaluate putative pathways in AAA formation, we induced Th1- or Th2-predominant cytokine environments in an inflammatory aortic lesion using murine aortic transplantation into WT hosts or those lacking the receptors for the hallmark Th1 cytokine IFN- $\gamma$, respectively. Allografts in WT recipients developed intimal hyperplasia, whereas allografts in IFN- $\gamma$ receptor-deficient (GRKO) hosts developed severe AAA formation associated with markedly increased levels of MMP-9 and MMP-12. Allografts in GRKO recipients treated with anti-IL-4 antibody to block the characteristic IL-4 Th2 cytokine or allografts in GRKO hosts also congenitally deficient in IL-4 did not develop AAA and likewise exhibited attenuated collagenolytic and elastolytic activities. These observations demonstrate an important dichotomy between cellular immune responses that induce IFN- $\gamma$ - or IL-4-dominated cytokine environments. The findings establish important regulatory roles for a Th1/Th2 cytokine balance in modulating matrix remodeling and have important implications for the pathophysiology of AAAs and arteriosclerosis.
\end{abstract}

\section{Introduction}

Abdominal aortic aneurysms (AAAs) occur in 5\% of individuals over the age of 50 and cause approximately 15,000 deaths annually due in large part to complications caused by rupture (1). Invasive procedures such as surgical or endovascular repair are currently the sole therapeutic options for this disease. Despite the demonstration of heightened expression of proinflammatory cytokines and elastolytic enzymes in human AAA lesions and certain risk factors associated with AAA disease, the mechanisms underlying AAA remain unknown, and lack of satisfactory animal models further hampers progress in understanding aneurysm formation.

End-stage AAAs exhibit chronic inflammation and excessive extracellular matrix (ECM) catabolism including degradation of both collagen and elastin $(2,3)$; matrix breakdown may thus be a primary mechanism underlying aneurysm development (4, 5). Recent studies confirmed that AAA tissue contains an excess of matrix-degrading proteinases, particularly members of the MMP family (4-6).

Cytokines regulate MMP expression in vivo and in vitro. Previous work showed elevated circulating levels of IL-1, IL-6, TNF- $\alpha$, and IFN- $\gamma$ in patients with AAA and also implicated these cytokines in AAA pathogenesis (7). In theory, such

Nonstandard abbreviations used: abdominal aortic aneurysm (AAA); BALB/c $(\mathrm{B} / \mathrm{c})$; bicinchoninic acid (BCA); double-deficient in IL-4 and IFN- receptor (DKO); extracellular matrix (ECM); IFN- $\gamma$ receptor-deficient (GRKO); T helper type 1 (Th1); tissue inhibitor of metalloproteinase (TIMP); tissue plasminogen activator (tPA); urokinase plasminogen activator (uPA)

Conflict of interest: The authors have declared that no conflict of interest exists.

Citation for this article: J. Clin. Invest. 114:300-308 (2004)

doi:10.1172/JCI200419855. proinflammatory mediators not only suppress the synthesis of procollagens I and III (8) but could also promote elastin and collagen degradation by activation of MMPs and suppression of tissue inhibitor of metalloproteinases (TIMPs). Moreover, MMPs can cleave TNF- precursor (pro-TNF- $\alpha$ ) (9) and pro-IL-1 $\beta$ (10) to yield their active forms, as well as release cytokines bound to the ECM (11), potentially perpetuating a vicious cycle that promotes aneurysm formation.

AAAs contain inflammatory cells including T cells, neutrophils, and macrophages, although it remains unclear whether these cells simply respond to the pathologic process or incite it. Most AAAs occur in the context of atherosclerosis, and atheromata of all stages contain $\mathrm{CD}^{+} \mathrm{T}$ lymphocytes bearing markers of both helper (CD4) and cytotoxic function (CD8) $(12,13)$. T cells and macrophages may affect atherogenesis by producing various cytokines that induce either matrix synthesis or degradation. In particular, different $\mathrm{T}$ cell subsets secrete IFN- $\gamma$ or IL-4 that characteristically drive opposing effects on a variety of biological processes. $\mathrm{CD}^{+} \mathrm{T}$ helper type 1 (Th1) cells and $\mathrm{CD} 8^{+} \mathrm{T}$ cytotoxic type 1 (Tc1) cells produce IFN- $\gamma$, IL- 2 , and TNF, whereas Th 2 and Tc2 cells secrete IL-4, IL-5, and IL-10. T cell responses polarize toward a Th1/Tc1 phenotype in the presence of IFN- $\gamma$, while IL-4 predisposes to Th2/Tc2 $\mathrm{T}$ cell responses (14).

Distinct patterns of cytokines produced by $\mathrm{T}$ cells might influence the clinical course of arterial disease. Indeed, previous work from our group showed that Th2 cytokines (IL-4, IL-5, or IL-10) and Th2-characteristic responses predominate in human AAA lesions. In contrast, Th1 responses predominate in stenotic atherosclerotic lesions (15). Although the mechanisms that underlie these observations remain unknown, it is noteworthy that in vitro, IL-4 stimulates the production of a variety of ECM proteins (16), while 
A

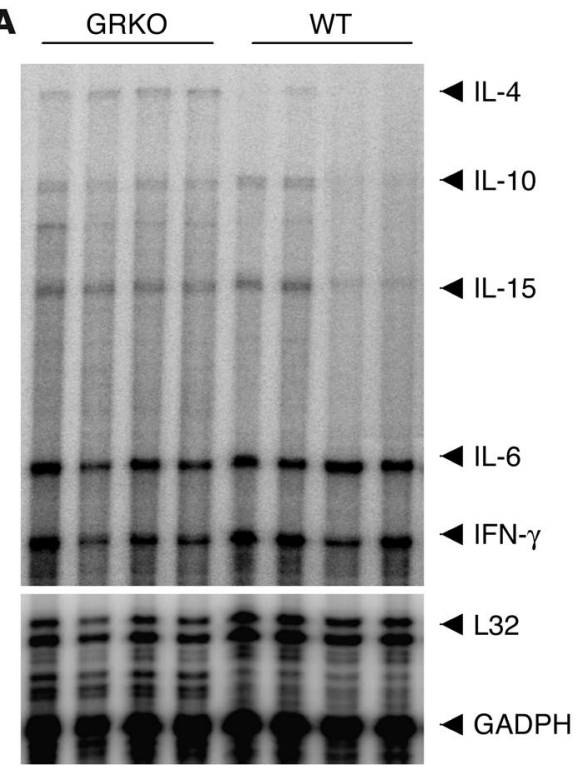

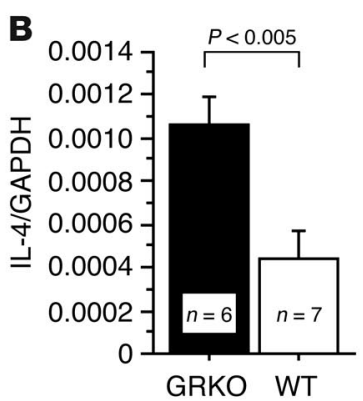

C

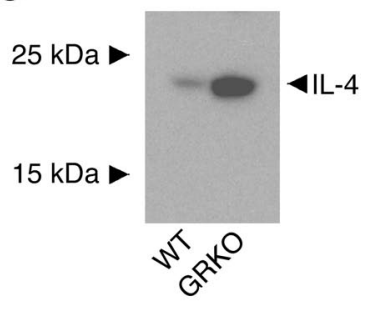

\section{Figure 1}

Aortic allografts from WT or GRKO recipients were harvested 1 week after transplantation and were analyzed for cytokine expression by RNase protection assay (RPA) (A and B) and Western blot for IL-4 (C). (A) Gel image of RPA. (B) Histogram of the optical densities from RPA data normalized to GAPDH and averaged for 6 or 7 grafts. (C) Protein extracts $(25 \mu \mathrm{g} / \mathrm{lane})$ of the aortic grafts of WT or GRKO recipients analyzed by Western blot for IL-4. blocking MMP-1 and MMP-9 release from alveolar macrophages without altering secretion of corresponding TIMPs (17).

This study used aortic allografts in IFN- $\gamma$ receptor-deficient (GRKO) mice as a novel immunologically driven model of aneurysm formation to test the hypothesis - derived from our observations in humans - that skewing to Th2-enriched environments induces AAA formation. We also explored the underlying molecular mechanisms that link Th1/Th2 balances to aneurysm formation.

\section{Results}

Allografts in WT and GRKO recipients have IFN- $\gamma$ - or IL-4-predominant cytokine environments, respectively. The aortic allograft model essentially allows the induction of a focal inflammatory response in discrete aortic segments. In WT combinations, this inflammation results in an intimal hyperplastic lesion without aneurysm formation (18). To test the role of specific cytokine subsets in the pathogenesis of AAA in this model, we induced either IFN- $\gamma$ - or IL-4dominant responses in transplanted BALB/c $\left(\mathrm{B} / \mathrm{c}, \mathrm{H}-2^{\mathrm{d}}\right)$ aortic segments in total allo-mismatched $129 \mathrm{SvEv}$ (129Sv, H-2 ${ }^{\mathrm{b}}$ ) hosts. Recipients were either WT, which resulted in predominant IFN- $\gamma$ production; or congenitally deficient in GRKO, which resulted in IL-4-predominant cytokine expression. Aortic allografts harvested one week after transplantation, at the peak of inflammatory cell infiltration (18), exhibited significantly greater IL-4 mRNA expression in the GRKO hosts compared with WT recipients (Figure 1, A and B). Other Th2 cytokine mRNAs (IL-10 and IL-15) were modestly increased in allografts in GRKO hosts compared with WT recipients (Figure 1A). Western blots (Figure 1C) showed the same

\section{Figure 2}

Aortic allografts from WT (A, C, E, and G) or GRKO (B, D, F, and H) recipients 12 weeks after transplantation. (A and $\mathbf{B}$ ) Representative gross appearance of aortas. ( $\mathbf{C}$ and $\mathbf{D})$ Echoaortograms. (E and $\mathbf{F}$ ) Histologic appearance of transverse sections. ( $\mathbf{G}$ and $\mathbf{H})$ Elastic van Gieson staining (EVG). Analysis of 9-10 aortic transplants from different donors yielded similar results. Control (WT) experiments, shown in panels A, C, E, and G, did not reveal measurable aneurysm formation or elastic tissue degradation. Arrowheads indicate elastic lamellae. marked skewing in IL-4 protein production in allografts recovered from GRKO versus WT recipients. Although intragraft IFN- $\gamma$ levels are comparable in WT and GRKO hosts, GRKO inflammatory cells cannot respond to IFN- $\gamma$ signals. Consequently, IL-4 largely governs responses to inflammation in GRKO hosts, whereas IFN- $\gamma$ responses direct inflammation in WT hosts.
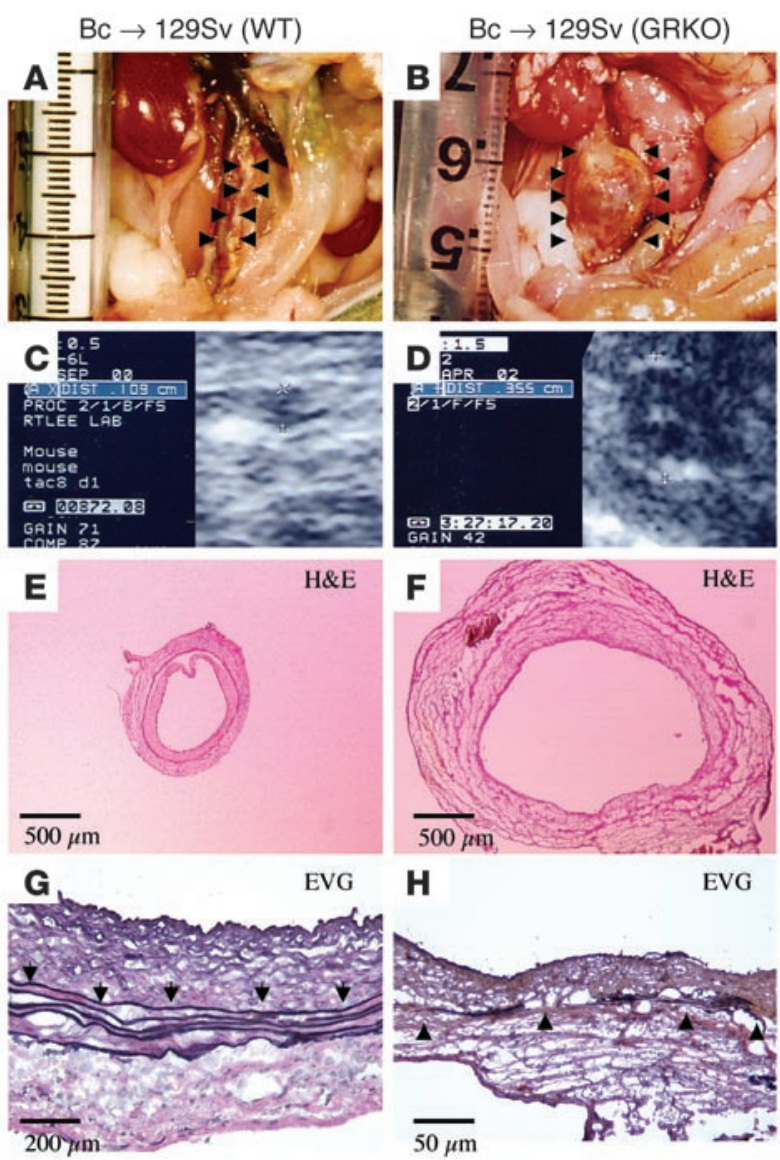

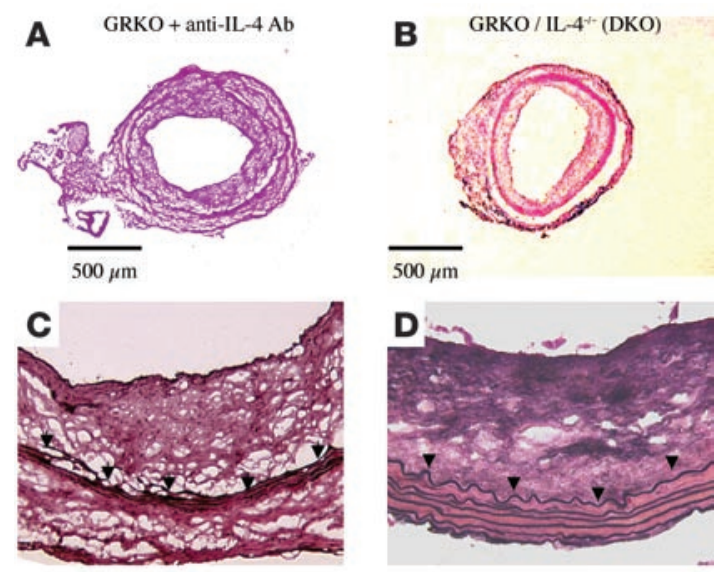

E

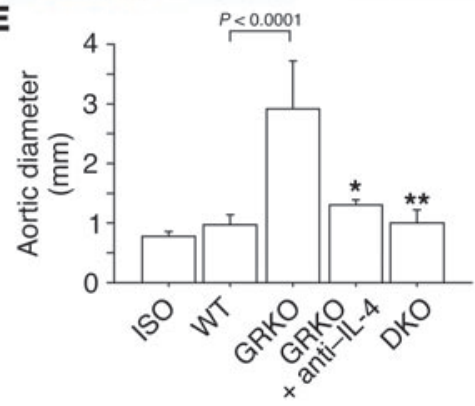

\section{Figure 3}

Aortic allografts from GRKO recipients treated with anti-IL-4 antibody (A and $\mathbf{C}$ ) or from DKO recipients (B and $\mathbf{D}$ ) harvested at 12 weeks after transplantation. (A and B) Representative histologic appearance of transverse sections. (C and D) Elastic van Gieson staining. (E) Analysis of aortic diameter from the isograft (Iso) $(n=5)$, WT recipient allografts $(n=9)$, GRKO recipient allografts $(n=10)$, allografts in GRKO recipients with anti-IL-4 antibody (11B11) treatment $(n=3)$, and DKO $(n=6)$ recipient allografts 12 weeks after transplantation. ${ }^{*} P<0.01$ vs. GRKO; ${ }^{* *} P<0.0001$ vs. GRKO.

Allografts in GRKO hosts develop aortic aneurysms. Lack of IFN- $\gamma$ receptor in the host resulted in profound aneurysm formation, in some cases leading to spontaneous rupture. Figure 2 shows the gross appearance (A and $B$ ), representative echoaortograms ( $\mathrm{C}$ and $\mathrm{D})$, and the histologic appearance (E and F, same magnification) of transverse sections of aortic allografts from WT (A, C, and E) or GRKO (B, D, and F) recipients 12 weeks after transplantation. The aortic diameter of the allografts of GRKO hosts was approximately three times larger than that of WT recipients. Aneurysmal allografts in GRKO hosts also showed markedly fragmented and attenuated elastic laminae in the aortic media (Figure $2 \mathrm{H}$ ) not seen in the WT recipient allografts (Figure 2G).

$I L-4$ blockade prevents aortic aneurysm formation in allografts in GRKO recipients. To examine specifically whether IL-4 blockade prevents aneurysm development, we treated GRKO recipients of $\mathrm{B} / \mathrm{c}$ aortic allografts with anti-IL-4 monoclonal antibody (11B11). GRKO hosts received $2 \mathrm{mg}$ of anti-IL-4 antibody by intraperitoneal injection 1 day before and 1 day after surgery, followed by $1 \mathrm{mg}$ antibody per week thereafter $(n=3)$. We also transplanted $\mathrm{B} / \mathrm{c}$ aortas into mice congenitally double deficient in IL-4 and IFN- $\gamma$ receptor (DKO, $n=6)$ and harvested the grafts 12 weeks after transplantation. Aneurysms did not develop in aortic allografts from GRKO recipients receiving anti-IL-4 anti- body (Figure 3A) or in allografts from DKO hosts (Figure 3B). Moreover, elastic van Gieson staining showed minimal elastic tissue fragmentation in grafts from GRKO hosts treated with 11B11 (Figure 3C) and no sign of elastolysis in grafts from DK O recipients (Figure $3 \mathrm{D}$ ).

Echoaortograms performed 12 weeks after transplantation and just before harvest showed in situ graft diameters of $0.8 \pm 0.1 \mathrm{~mm}$ in isografts $(n=5) ; 1.0 \pm 0.2 \mathrm{~mm}$ in WT hosts $(n=9 ; P=\mathrm{NS}$ vs. isografts); $2.9 \pm 0.8 \mathrm{~mm}$ in GRKO hosts $(n=10 ; P<0.0001$ vs. WT); $1.3 \pm 0.1 \mathrm{~mm}$ in GRKO hosts receiving anti-IL-4 treatment $(n=3 ; P=0.005$ vs. GRKO); and $1.0 \pm 0.2 \mathrm{~mm}$ in DKO hosts $(n=6$; $P<0.0001$ vs. GRKO). Our results indicate that the presence of IL-4 predominance induces aortic aneurysm formation (Figure 3E) and that IL-4 blockade prevents aneurysm formation.

Aneurysm formation does not result from increased blood pressure. To examine whether IL-4 elaboration or blockade affected systemic blood pressure (and thereby influenced AAA formation), we measured the recipient systolic arterial pressure 12 weeks after transplantation by a tail cuff. Blood pressure in all transplant groups was similar: $106 \pm 18 \mathrm{mmHg}$ in WT; $101 \pm 13 \mathrm{mmHg}$ in GRKO; and $96 \pm 11 \mathrm{mmHg}$ in DKO $(P=\mathrm{NS})$.

Increased $m R N A$ expression of elastolytic MMPs $m R N A$ correlates with allograft aneurysm formation in GRKO recipients. The ECM protein elastin consists of highly cross-linked, hydrophobic tropoelastin monomers that confer resilience to the elastic fibers. The hydrophobicity and extensive cross-linking of tropoelastin result in an insoluble fiber that resists proteolysis. Under normal conditions, elastin has minimal turnover (19). Nevertheless, destruction of elastic fibers characterizes certain pathological situations such as AAAs (20).

The ability of certain MMPs, e.g., MMP-2, -3 , -9 , and -12 , to degrade elastin may have particular relevance to AAA formation, as human AAAs exhibit abundant MMP-2 and MMP -9 expression $(21,22)$ and, in some cases, excessive MMP-1 and MMP-3 (4-6). In experimental models, targeted disruption of MMP-9 results in decreased elastin fiber degradation after elastase perfusion in mouse aortas and suppresses the subsequent development of aortic aneurysms (23). The other elastolytic MMPs such as matrilysin (MMP-7) (24) and macrophage metalloelastase (MMP-12) $(25,26)$ may play even greater roles in very large aneurysms.

In addition to the MMPs, AAAs contain excessive serine proteases such as plasmin, as well as elevated plasminogen activators (PAs) (i.e., urokinase PA, uPA; and tissue PA, tPA), compared with normal aortic tissue $(4,5)$. Cysteine proteinases such as cathepsin-L, $-\mathrm{S}$, and -K or serine proteinases such as neutrophil elastases have elastinolytic potential (27) and may also contribute to AAA formation.

To identify which elastinolytic or collagenolytic enzymes contribute to elastin degradation and AAA formation in grafts in GRKO mouse hosts, we performed LightCycler-based real-time PCR using cDNA prepared from total RNA extracted from aortic allografts one week after transplant at the peak of inflammatory cell infiltration (18). We examined MMP-1 through MMP-24, TIMP-1 through TIMP-4, cathepsin-L, -S, and -K, neutrophil elastase, uPA, and tPA. MMP-2, $-3,-9,-12,-13,-15$, and -19 mRNA expression all increased significantly in allografts from GRKO recipients with parallel increases in TIMP-1, TIMP-2, and TIMP-3 compared with grafts in either WT or DKO hosts $(n=6$ for each; Figure 4). Allografts from DKO had significantly lower MMP-10 mRNA levels than allografts from GRKO recipients; 

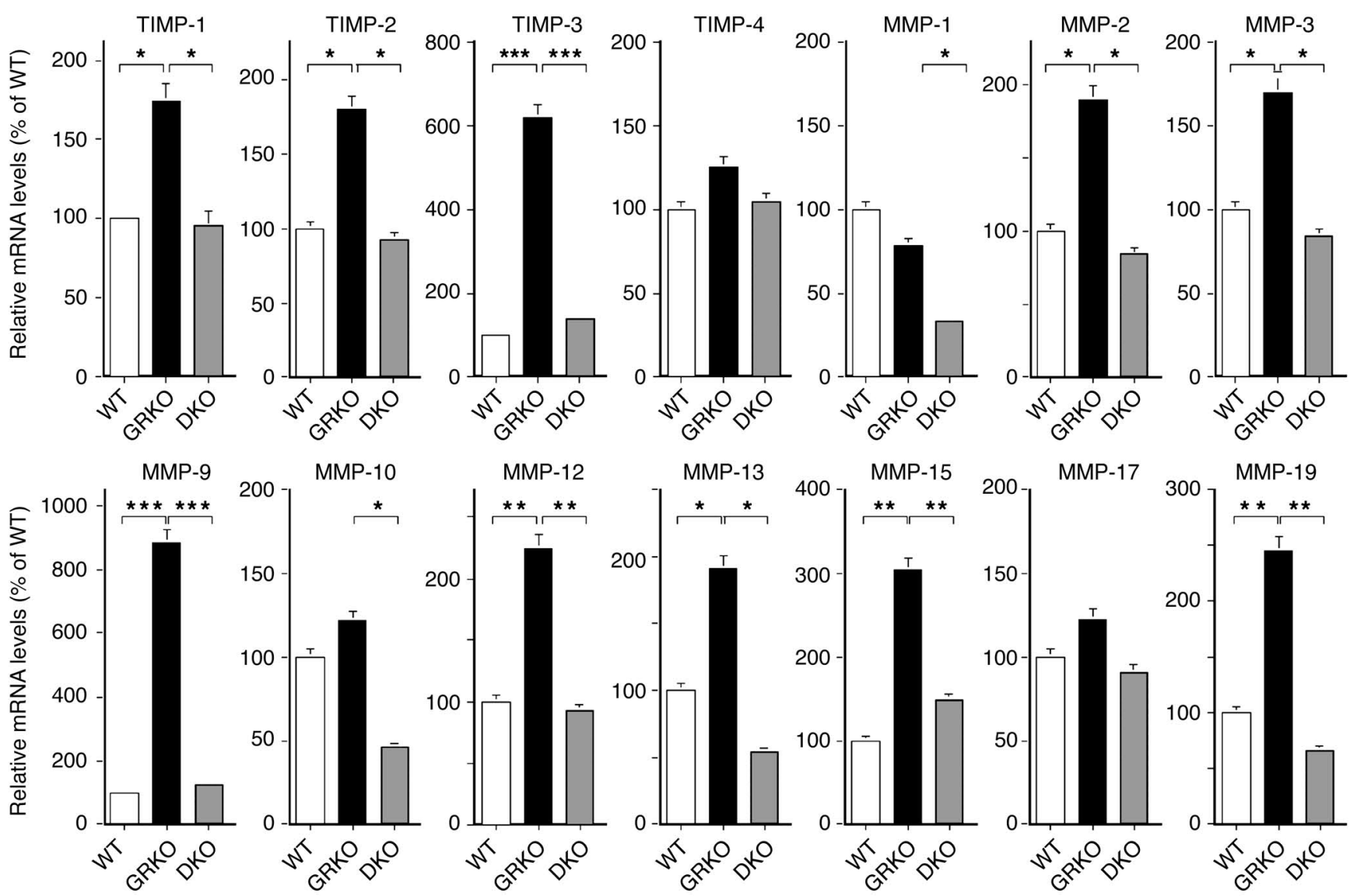

Figure 4

Metalloproteinase expression during AAA development. The MMP mRNA levels of aortic allografts from WT (white bars), GRKO (black bars), or DKO (gray bars) recipients ( $n=6$, each). Total RNA was extracted from the aortic allografts and first-strand cDNA generated by reverse transcription was subjected to real-time quantitative PCR with the LightCycler. Data represent the mean \pm SEM of six determinations of the percentage of mRNA copies relative to untreated growing cells. ${ }^{\star} P<0.05 ;{ }^{\star \star} P<0.01 ;{ }^{* \star \star} P<0.001$.

expression of mRNA encoding the other proteases did not differ between groups (data not shown).

Elevated enzymatic activities for MMP-12 in allografts in GRKO hosts. To test formally whether the elevated MMP mRNA resulted in increased functional enzymatic activity, we performed Western blot analysis, elastase colorimetric assay, and zymography. Western blot analysis demonstrated markedly increased expression of MMP-12 in allografts from GRKO hosts compared with allografts from WT and DKO recipients (Figure 5A). At one week, aortic allografts in GRKO recipients had significantly greater elastase activity $(6.61 \pm 1.60 \mu \mathrm{U} / \mathrm{g} ; n=6)$ relative to WT hosts $(1.75 \pm 0.41 \mu \mathrm{U} / \mathrm{g} ; n=6 ; P<0.0001)$ and $\mathrm{DKO}$ recipients $(0.61 \pm 0.15 \mu \mathrm{U} / \mathrm{g} ; n=6 ; P<0.0001)$. Immunoprecipitation with anti-MMP-12 antibody of the proteins recovered from allografts in GRKO hosts removed the majority of the elastolytic activity with residual elastase colorimetric assay activity of $0.04 \pm 0.05$ $\mu \mathrm{U} / \mathrm{g}(P<0.0001 \mathrm{vs}$. nonimmunoprecipitated proteins from aortic grafts in GRKO hosts; $n=6$; Figure 5B). Gelatin zymography (Figure 5C) and casein zymography (Figure 5D) corroborated these results; increased activities corresponded to MMP-2, MMP-9, and MMP-12 proteases, as indicated. The same amounts of proteins loaded in the gels suggests much higher MMP-12 activities than MMP-9 in GRKO recipient allografts.
The majority of the graft-infiltrating cells during aneurysm formation are macrophages. A number of cell types including endothelial cells, medial smooth muscle cells, and adventitial connective tissue cells might contribute to the local pool of MMPs in vascular tissues (28). Monocyte-derived macrophages in particular can express abundant active MMPs (4, 5, 29), especially MMP-9 and MMP-12 in diseased tissues. MMP activity depends on control at the transcriptional level by cytokines, on activation of the proenzyme forms of the MMPs, and on the local concentrations of TIMPs $(28,30)$. We observed medial elastinolysis in the allografts in GRKO hosts within 1-4 weeks of transplantation (18), when the majority of the graft-infiltrating cells consist of

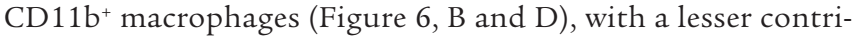
bution of $\mathrm{CD}^{+} \mathrm{T}$ cells (Figure 6, A and $\mathrm{C}$ ) and $\mathrm{CD}^{+} \mathrm{T}$ cells (data not shown). Immunoreactive MMP-12 (Figure 6, F and H) colocalized predominantly with $\mathrm{CD}_{11} \mathrm{~b}^{+}$cells (Figure 6, E and $\mathrm{G})$, which suggests that infiltrating macrophages provide much of the MMP-12 in developing AAA.

$I L-4$ angments and IFN- $\gamma$ diminishes MMP-12 mRNA expression in macrophages. To examine whether IL- 4 and IFN- $\gamma$ can directly modulate MMP-12 expression in macrophages, we cultured bone marrow-derived macrophages from WT and GRKO mice in the presence of IL-4, IFN- $\gamma$, and/or anti-IL-4 antibody. Both conventional 
A

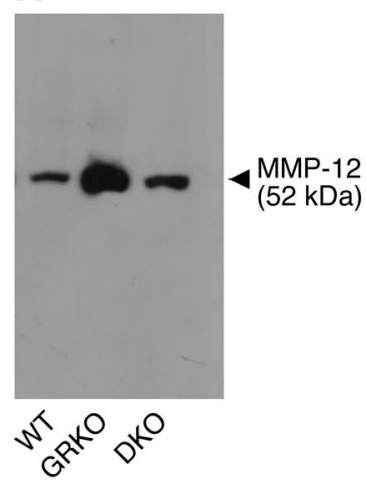

B

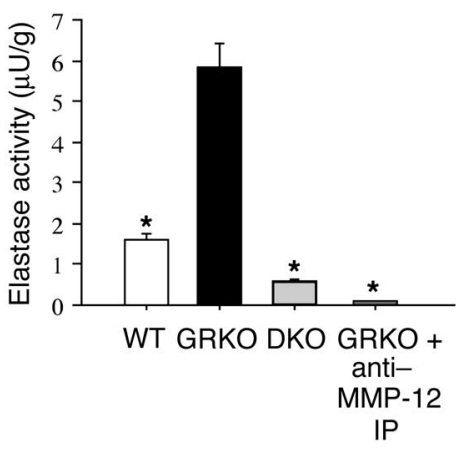

C

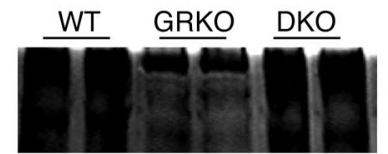

MMP-9 (92 kDa)

MMP-9 $(82 \mathrm{kDa})$

4 MMP-2 (62 kDa)

D

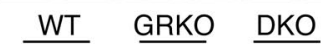

$4 \mathrm{MMP}-12(20 \mathrm{kDa}$, active)

PCR (23 cycles) with subsequent gel electrophoresis (Figure 7A) and real-time quantification of MMP-12 gene copy number using LightCycler (Figure 7B) were performed on cDNA from total RNA extracted after 18 hours of macrophage culture. IL-4 increased MMP-12 mRNA expression, and anti-IL-4 antibody abolished this effect. Moreover, IFN- $\gamma$ decreased IL-4-induced MMP-12 mRNA expression in WT macrophages but did not inhibit MMP-12 expression in GRKO macrophages (data not shown). MMP-9 mRNA expression exhibited the same responses to IFN- $\gamma$ and IL-4 as MMP-12 (data not shown).

\section{Discussion}

This study sought to examine the relative effects on arterial disease of a prototypical "proinflammatory cytokine" (e.g., IFN- $\gamma$ from Th1 cells) versus an "anti-inflammatory cytokine" (e.g., IL-4 from Th2 cells). This study showed that IL-4-driven inflammation in aortic transplants induced severe AAA formation associated with markedly increased expression of MMP-9 and MMP -12; blockade of IL-4 effects via anti-IL-4 or congenital absence of IL-4 reduced AAA formation with associated decreases in elastic tissue fragmentation and in MMP-9 and MMP-12 expression.

Previous work found elevated proinflammatory cytokines or chemokines (e.g., IL-1, IL-6, IL-8, TNF- $\alpha$, IFN- $\gamma$, monocyte chemoattractant protein-1 [MCP-1]) systemically or in human

\section{Figure 6}

Representative immunohistochemistry of $\mathrm{CD}_{4}^{+}(\mathbf{A}$ and $\mathbf{C})$ and $\mathrm{CD} 11 \mathrm{~b}^{+}$ ( $B$ and $\mathbf{D}$ ) cells in allografts from GRKO recipients harvested 4 weeks after transplantation $(n=6)$. Boxed area in $\mathbf{A}$ or $\mathbf{B}$ (original magnification, $\times 100$ ) is enlarged in $\mathbf{C}$ or $\mathbf{D}$ (original magnification, $\times 400$ ), respectively. The majority of the graft infiltrating cells, especially adjacent to degraded elastic lamellae, consisted of CD11 b+ macrophages. Expression of MMP-12 ( $\mathbf{F}$ and $\mathbf{H}$ ) colocalized with CD11 b+ cells ( $\mathbf{E}$ and $\mathbf{G}$ ) in allografts from GRKO recipients harvested 4 weeks after transplantation $(n=6)$.

\section{Figure 5}

Western blot for MMP-12, elastase colorimetric assay, and gelatin and casein zymography. (A) Representative gel image of Western blot analysis. Protein extracts $(20 \mu \mathrm{g} /$ lane) of the aortic grafts of WT $(n=6)$, GRKO $(n=6)$, or DKO $(n=6)$ recipients analyzed by Western blot for MMP-12. The gel images represent qualitatively similar results. (B) The elastase colorimetric assay shows significantly greater elastase activity in the proteins extracted from allografts in GRKO hosts $(n=6)$ compared with proteins extracted from allografts in WT $(n=6)$ or DKO $(n=6)$ hosts. After anti-MMP-12 immunoprecipitation (IP) $(n=6)$, the proteins from allografts from GRKO recipients had significantly reduced elastase activity, which indicates that the majority of elastolytic activity in those allografts derives from MMP-12. Bar shows mean \pm SEM; ${ }^{*} P<0.0001$. (C and D) Representative gel images of gelatin zymogram (C) and casein zymogram (D). Protein extracts $(20 \mu \mathrm{g} / \mathrm{lane})$ of the aortic grafts of WT $(n=6)$, GRKO $(n=6)$, or DKO $(n=6)$ recipients analyzed by (C) gelatin- or (D) casein-zymogram for MMPs. The gel images represent qualitatively similar results. We could detect only 92 $\mathrm{kDa}$ and $72 \mathrm{kDa}$ active bands from GRKO recipient allografts in the gelatin zymogram (C) and only $20 \mathrm{kDa}$ active band from GRKO recipient allografts in the casein zymogram (D).

AAA tissue obtained at surgery, implicating these cytokines in AAA pathogenesis (7). Atherosclerotic lesions frequently associate with AAAs, and similar risk factors for atherosclerosis and AAA formation have been identified. However, differences in proteolytic activities and disparities in the characteristics of patients that develop AAA versus stenotic lesions suggest that AAA formation does not necessarily require preexisting atherosclerosis (31). Moreover, our previous work showed that Th2 cytokines and responses predominated in human AAA lesions, while Th1 responses predominated in stenotic atherosclerotic lesions (15).
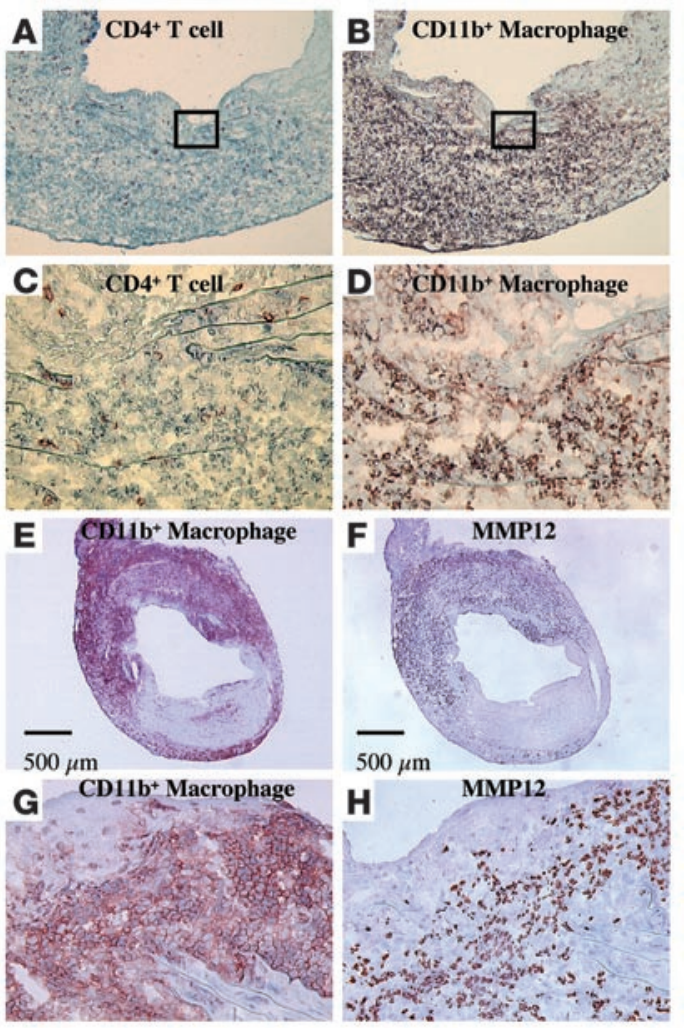


\section{Conventional PCR}

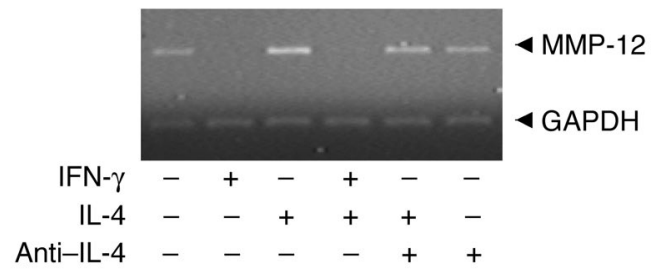

B

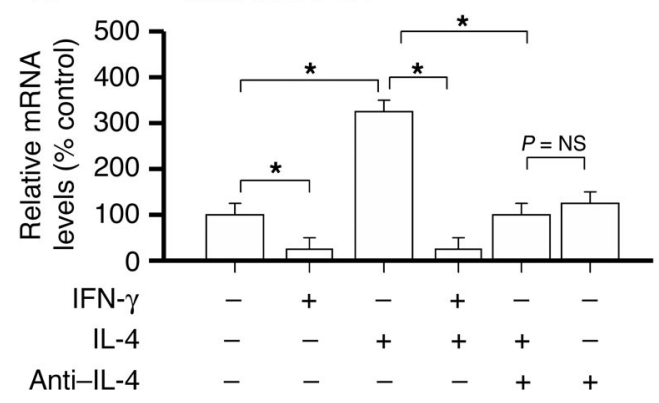

Figure 7

Conventional RT-PCR (A) and real-time PCR (B) for MMP-12 from WT bone marrow-derived macrophages incubated 18 hours with IL-4 (10 $\mathrm{ng} / \mathrm{ml})$, IFN- $\gamma(500 \mathrm{U} / \mathrm{ml})$, and/or anti-IL-4 antibody $(5 \mu \mathrm{g} / \mathrm{ml}, 11 \mathrm{~B} 11)$. IL-4 augmented and IFN- $\gamma$ inhibited MMP-12 mRNA expression of the WT macrophages. In addition, 11B11 inhibited IL-4-induced augmentation of MMP-12 mRNA expression by the WT macrophages. Bar shows mean $\pm \operatorname{SEM}\left(n=6\right.$, each). ${ }^{*} P<0.0001$.

Elastase perfusion (23), angiotensin II infusion (32), or local treatment with $\mathrm{CaCl}_{2}$ (33) result in aneurysm formation in animals. However, no prior studies have used animals with specific chemokine or cytokine deficiencies to define the function of these mediator proteins in aneurysm formation.

The current study used allo-mismatched aortic transplantation to induce local inflammation in aortic segments with WT or GRKO animals to elicit IFN- $\boldsymbol{\gamma}$ - or IL-4-predominant responses, respectively. Death of medial smooth muscle cells per se does not cause aneurysm formation (18). Although acute rejection causes virtually complete medial smooth muscle cell death in allo-mismatched WT recipient aortic allografts, they do not develop aneurysms and in fact develop intimal hyperplasia (18). Strikingly, GRKO recipient allografts developed profound aortic aneurysms with degradation of the medial elastic laminae similar to that seen in human AAAs. These results demonstrate in vivo that specific blockade of IFN- $\gamma$ signaling (Th1 depletion) and/or augmented IL-4 (Th2 predominance) can directly modulate elastolytic enzymes and yield degradation of elastic laminae of the arterial tunica media.

In humans, aneurysms can form because of atherosclerosis, focal loss of elastic and smooth muscle fibers (cystic medial degeneration, e.g., due to hypertension and Marfan syndrome), congenital defects in the vascular wall (e.g., berry aneurysms), and/or infections (e.g., luetic aneurysms). The pathologic characteristics of AAA classically consist of inflammatory infiltrates with elastin and collagen (types I and III) degradation, resulting in vessel wall expansion and potentially rupture. All types of AAAs require degradation or loss of elastic fibers in the media. Perturbations in either synthesis or degradation could conceivably diminish the elastin and collagen fibers. However, mRNA levels of procollagen and tropoelastin from aortic allografts one week after transplant showed no differences in expression among WT, GRKO, and DKO recipient allografts (data not shown).

MMP-9 and MMP-12 degrade elastin efficiently, and human AAA lesions contain these MMPs, as well as MMP-1, MMP-3, and MMP-13. Activation of latent MMPs requires proteolytic cleavage of a region that unmasks the active zinc-containing catalytic site. In addition, MMP activity also depends on the level of endogenous inhibitors such as TIMPs. Serine proteases such as plasmin, uPA, and tPA also localize in AAAs and correlate with macrophage infiltration (34). Plasmin and UPA activate MMP-3, MMP-12, and MMP-13, and since absence of uPA in mice protects from rupture of aortic aneurysms, uPA may regulate the downstream cascade of MMP effectors (35).

This study used real-time PCR with cDNA extracted from the early stages of aortic allografts with or without AAA to assess mRNAs that encode multiple MMPs and elastolytic enzymes. We also measured the elastolytic activities using protein extracted from the AAA tissue. The results suggest that relatively few MMPs contribute to aortic expansion under these conditions. Increased TIMP-1, TIMP-2, and TIMP-3 mRNA expression paralleled MMP-9, MMP-12, and MMP-13 mRNA elevation, with higher mRNA expression in GRKO allografts and lower mRNA expression in DKO allografts. Thus, increased TIMP activity alone does not suffice to modulate MMP degradation. Allografts in GRKO hosts showed markedly higher elastolytic activities relative to aortic grafts in WT and DKO recipients, and this elastolytic activity diminished markedly after MMP-12 immunoprecipitation.

Th2 cytokines are usually considered largely anti-inflammatory, although Th2-dominated inflammatory responses clearly drive allergic asthma, as well as some proinflammatory activity in other in vivo models (36). While IL-4 suppresses some MMP protein expression in monocytes (37), other reports demonstrated that IL-13 (originally described as an IL-4-like cytokine) potently induces MMP-2, -9, -12, -13, and -14 (38). We also previously reported an apparently paradoxical role of IL-10 in exacerbating allograft arteriosclerosis (39). The current work clearly supports a role for IL-4 in increasing selected elastolytic activities. At the same time, this study shows that IFN- $\gamma$ can protect against augmented elastase activities that induces aneurysm formation. Indeed, we found that total allo-mismatched aortic allografts of IFN- $\gamma$-deficient recipients developed aneurysms to the same extent as allografts in GRKO recipients (data not shown).

Various cytokines differentially affect protease activity. Thus, earlier studies demonstrated an important role for MMP-9 in AAA development and implicated proinflammatory (Th1) cytokines in elevating macrophage MMP expression. Lungspecific IFN- $\gamma$ overexpression in transgenic mice also induced MMP-12 and stimulated the release of MMP-9, leading to pulmonary emphysema (40). However, IFN- $\gamma$ has inhibitory effects on MMP-9 in human monocytes and macrophages (41) and in murine peritoneal macrophages (42), as well as on MMP-12 in murine macrophages (43).

Atherosclerosis is also a major risk factor for AAA formation, and IFN- $\gamma$ potentiates atherosclerosis (44); moreover, IFN- $\gamma$ is necessary for inducing transplant-associated arteriosclerosis (45). The current study contradicts earlier results suggesting that IFN- $\gamma$ is important for AAA development. However, in those models, atherosclerosis requires inflammatory cell recruitment, 
and in the absence of IFN- $\gamma$, this inflammation is reduced. In the current work, we use an alloresponse to drive the local inflammatory cell recruitment and can do so while formally testing the relative roles of Th1 and Th2 cytokines. Although some of the differences between atherosclerosis and alloresponse AAA development may be due to the specific models, it is noteworthy that current results mirror the cytokine profiles described in human AAAs (15). We therefore believe that IFN- $\gamma$ is capable of attenuating AAA lesions.

While the model used here involved major histocompatibility mismatched allografts, there appears to be a striking similarity between our results and recent work on viral infections in large elastic arteries; increased transmural arterial inflammation occurs in GRKO mice infected with CMV (46) or $\gamma$-herpesvirus 68 (47), suggesting that the elastic media of great vessels may be an immune-privileged site, perhaps with privilege preserved in part by IFN- $\gamma$. The current study focused on Th1/Th2 cytokine milieu on AAA using allogeneic responses based on our previous findings of Th2-predominant cytokine expression in the human AAA lesions and did not explore the effects of atherosclerosis generally observed in human AAA lesions. It is possible that human atherosclerotic lesions need some additional (perhaps idiosyncratic) Th2-predominant inflammatory responses to develop aneurysm formation rather than Th1-dominated obstructive lesions. Further study remains to elucidate such triggers.

This study demonstrates that blockade of IFN- $\gamma$ signaling pathways and subsequent IL-4-mediated events induced AAA formation associated with augmented elastolytic activity primarily due to increased MMP-12 expression. The majority of inflammatory cells at the early stage of AAA consist of macrophages that also express MMP-12 as shown by immunohistochemical staining. In addition, in vitro macrophage cultures show augmented expression of elastolytic MMP-12 in the presence of IL-4, an effect inhibited by coadministration of IFN- $\gamma$. These findings identify IL-4 as an important stimulus to AAA formation and suggest that IFN- $\gamma$ can potentially act to attenuate collagenolytic and elastolytic activity. Our results furnish new insight into the mechanisms of aneurysmal disease and suggest that IL-4 blockade or IFN- $\gamma$ activation may inhibit the formation and/or expansion of arterial aneurysms.

\section{Methods}

Mice. The studies reported here conform to the principles outlined by the Animal Welfare Act and the NIH guidelines for the care and use of animals in biomedical research. WT 129SvEv and B/c mice were obtained from Taconic Farms Inc. (Germantown, New York, USA). 129Sv(GRKO) mice were obtained from The Jackson Laboratory (Bar Harbor, Maine, USA). The generation and the basic characteristics of the mutant mouse strain (129SvEv) with a disruption in the gene encoding the $\alpha$-chain of the IFN- $\gamma$ receptor have been described (48). C57BL/6 IL-4-deficient mice were obtained from The Jackson Laboratories and crossed to GRKO mice to obtain DKO mice (back-crossed onto 129Sv, for at least 4 generations). The mice were maintained in the Harvard Medical School animal facilities on acidified water. Sentinel animals in the same room were surveyed serologically and were consistently negative for all viral pathogens.

Aortic transplantation. WT B/c, WT 129Sv, and 129Sv(GRKO) male mice aged 8-12 weeks and weighing 20-25 g were used as recipients or donors. Aortic transplantation was performed as described (18).
No immunosuppressive medication was used. Grafts that became occluded within the first 10 days were considered technical failures and were excluded from further consideration. The graft success rate was more than $90 \%$.

Noninvasive blood pressure measurements. Blood pressure and heart rate were measured noninvasively using the Visitech BP-2000 Blood Pressure Analysis System (Visitech Systems, Apex, North Carolina, USA). To ensure accuracy and reproducibility, the mice were trained for 1 week prior to the experiment, and measurements were taken at the same time each day. Unanesthetized mice were placed on the specimen platform, and their tails were placed through tail cuffs and secured in place with tape. Following a 15minute warm-up period, 10 preliminary cycles were performed to allow the mice to adjust to the inflating cuff. For each mouse, 10 cycles were recorded for each time point, and the data for each group of mice were combined.

Quantification of $m R N A$ using LightCycler-based real-time, quantitative RT-PCR. Messenger RNA levels were quantitated for a variety of MMPs from aortic allografts harvested one week after transplantation or from in vitro-cultured marrow-derived macrophages using LightCycler-based real-time PCR and quantitative RT-PCR protocols using the LightCycler-DNA Master SYBR Green I kit (Roche Molecular Biochemicals, Mannheim, Germany), as described previously (49). Total RNA was extracted from aortic grafts using Trizol (Invitrogen Corp., Carlsbad, California, USA) and purified with RNeasy kit (Qiagen Inc., Valencia, California, USA); cDNA was synthesized with a First-Strand cDNA Synthesis Kit followed by DNase treatment (Invitrogen). TaqStart antibody (CLONTECH, Palo Alto, California, USA) was used to prevent generation of nonspecific amplification products. Quantitation was performed using primers designed by Primer3 program (www.genome.wi.mit.edu/cgi-bin/primer/primer3_www.cgi), and PCR reactions were examined by agarose gel electrophoresis to confirm melting curve results. We devised methods suitable for quantitation of the following mRNA: MMP-1, -2, -3, $-7,-9,-12,-13,-15,-17$, and -19; TIMP-1, $-2,-3$, and -4 ; neutrophil elastase; cathepsins-L, -S, and -K; uPA; and tPA. Fluorescence data were quantitatively analyzed using serially diluted control samples to produce standard curves. Data represent the mean \pm SEM of six to seven determinations of the percentage of mRNA copies relative to the control WT recipient allografts, which was set to $100 \%$.

Immunoblotting. Aortic grafts from WT $(n=6)$, GRKO $(n=6)$, and DKO $(n=6)$ recipients were harvested and homogenized in 50 $\mathrm{mmol} / 1$ Tris/HCL (Sigma-Aldrich, St. Louis, Missouri, USA) ( $\mathrm{pH}$ 8.0 ) containing $0.5 \%$ Triton-X100 (Sigma-Aldrich) and centrifuged $\left(500 \mathrm{~g}\right.$ for 30 minutes at $\left.4^{\circ} \mathrm{C}\right)$. Samples were centrifuged at $3,000 \mathrm{~g}$ for 30 minutes, and the protein concentration for each tissue extract was determined using the bicinchoninic acid (BCA) assay (Pierce, Rockford, Illinois, USA). Total proteins $(20 \mu \mathrm{g} /$ well $)$ were separated by standard SDS-PAGE and blotted to polyvinylidene difluoride membranes (Bio-Rad, Hercules, California, USA) using a semidry blotting apparatus $\left(3 \mathrm{~mA} / \mathrm{cm}^{2}, 60 \mathrm{~min}\right.$; Bio-Rad). Blots were blocked, and primary and secondary antibodies were diluted in $5 \%$ defatted dry milk/PBS/0.1\% Tween 20 (Sigma-Aldrich). Primary rabbit anti-mouse MMP-12 antibodies (Santa Cruz Biotechnology Inc., Santa Cruz, California, USA) were applied at $2 \mu \mathrm{g} / \mathrm{ml}$. After 1 hour of incubation with the primary antibody, blots were washed 3 times (PBS/0.1\% Tween 20) and the relevant secondary, peroxidase-conjugated antibody $(500 \mathrm{ng} / \mathrm{ml}$, Jackson Immunoresearch, West Grove, Pennsylvania, USA) was added for another hour. Finally, the blots were washed (20 min, PBS/0.1\% Tween 20) and immunoreactive 
proteins visualized using the SuperSignal West Femto kit (Pierce). Densitometric analysis of immunoreactive bands employed ImagePro software (Media Cybernetics, Silver Spring, Maryland, USA) applied to digital images of the Western blots. We performed quantitative immunoblots using equal starting amounts of protein.

Zymography. Aortic grafts from WT $(n=6), \operatorname{GRKO}(n=6)$, and DKO $(n=6)$ recipients were harvested and homogenized in $50 \mathrm{mmol} / \mathrm{l}$ Tris/HCL ( $\mathrm{pH} 8.0$ ) containing $0.5 \%$ Triton-X100 and centrifuged $\left(500 \mathrm{~g}\right.$ for 30 minutes at $\left.4^{\circ} \mathrm{C}\right)$. Samples were centrifuged at $3,000 \mathrm{~g}$ for 30 minutes, and the protein concentration for each tissue extract was determined using the BCA assay (Pierce). Total proteins $(20 \mu \mathrm{g} /$ well) were separated under nonreducing conditions by SDS-PAGE containing $1 \mathrm{mg} / \mathrm{ml}$ gelatin or casein (Bio-Rad). After electrophoresis, the proteins were renatured by soaking the gels in renaturation buffer (Bio-Rad). Gels were stained with Coomassie brilliant blue R250 and then destained in 25\% methanol/20\% acetic acid.

Colorimetric assay of elastase. The same protein samples as prepared for immunoblotting were used for enzymatic analysis by colorimetry. The elastase assay is based on the specific hydrolysis of MeOSuc-Ala-Ala-Pro-Val-p-nitroanilide (Calbiochem, San Diego, California, USA) by elastase, resulting in the release of the $p$-nitroaniline ( $p \mathrm{NA}$ ) moiety (50). Each $20 \mu \mathrm{g}$ of sample was incubated with $20 \mu \mathrm{g}$ of substrate on a 96-well flat-bottom microplate (Corning Inc., New York, New York, USA) at $37^{\circ} \mathrm{C}$ for 60 minutes, and released $p \mathrm{NA}$ (emission at $405 \mathrm{~nm}$ ) was measured. The activity of the samples was calculated relative to an elastase type III (4 U/mg, Sigma-Aldrich) standard curve.

We also performed elastase colorimetric assays using the protein samples from GRKO recipient allografts following anti-MMP-12 immunoprecipitation. The protein samples of the GRKO recipient allografts were incubated with anti-MMP-12 antibody $(2 \mu \mathrm{g} / \mathrm{ml})$ for 18 hours at $4^{\circ} \mathrm{C}$, followed by incubation with immobilized protein $\mathrm{G}$ for 30 minutes at $4^{\circ} \mathrm{C}$ using an Immunoprecipitation Kit (Pierce). Samples were centrifuged, and the buffer was collected and adjusted to $\mathrm{pH}$ 8.0. The protein concentration was normalized using the BCA assay before the elastase assay.

Immunohistochemistry. Immunohistochemistry was performed to evaluate the relative percentages of CD4-, CD8-, and CD11b-positive inflammatory cells in the cardiac allografts as described pre- viously (51). For MMP-12 staining, $5 \mu \mathrm{m}$ cryosections were fixed in $5 \%$ paraformaldehyde before preincubation with $0.5 \% \mathrm{H}_{2} \mathrm{O}_{2}$. Slides were incubated with $10 \%$ normal goat serum and stained with purified monoclonal anti-MMP-12 antibodies $(2 \mu \mathrm{g} / \mathrm{ml})$ or negative control antisera (rabbit IgG, $2 \mu \mathrm{g} / \mathrm{ml}$, BD Biosciences - Pharmingen, San Diego, California, USA). Sections were incubated with biotinylated goat anti-rabbit IgG antibodies $(1 \mu \mathrm{g} / \mathrm{ml}$, Southern Biotechnology Associates Inc., Birmingham, Alabama, USA), followed by streptavidin-peroxidase (DAKO, Carpinteria, California, USA). Antibody binding was visualized with 3-amino9-ethyl carbazole (DAKO), and nuclei were counterstained with Gill's hematoxylin (Sigma-Aldrich).

Statistical analysis. Comparisons between groups was performed using a two-way ANOVA with the post-hoc test. Since the AAA lesion area was not normally distributed, the Mann-Whitney $U$ test was used to evaluate differences in aortic size. Statview 4.5 for Macintosh (Abacus Concepts Inc., Berkeley, California, USA) was used for all statistical calculations.

\section{Acknowledgments}

This study was supported by NIH grants RO1 HL-43364 (to P. Libby and R.N. Mitchell), GM-67049 (to R.N. Mitchell and K. Shimizu), and HL-67283 (to K. Shimizu and R.N. Mitchell) and a Roche Organ Transplantation Research Foundation grant award (K. Shimizu), a Harvard Medical School BWH (Brigham and Women's Hospital) Fellowship Award (K. Shimizu), and a Grant-in-Aid for Scientific Research from the Ministry of Education, Culture, Sports, Science, and Technology of Japan (to M. Shichiri). We thank E. Shvartz, J. Allen, and E. Simon-Morrissey for their technical expertise and K. Williams for her editorial expertise.

Received for publication August 20, 2003, and accepted in revised form May 4, 2004.

Address correspondence to: Koichi Shimizu, Cardiovascular Division, Department of Medicine, Brigham and Women's Hospital, Harvard Medical School, 77 Avenue Louis Pasteur, NRB7, Boston, Massachusetts 02115, USA. Phone: (617) 525-4556; Fax: (617) 525-4380; E-mail: ksmz@rics.bwh.harvard.edu.
1. Hallett, J.W., Jr. 2000. Management of abdominal aortic aneurysms. Mayo Clin. Proc. 75:395-399.

2. Glagov, S. 1977. Morphology of collagen and elastin fibers in atherosclerotic lesions. Adv. Exp. Med. Biol. 82:767-773.

3. Campa, J.S., Greenhalgh, R.M., and Powell, J.T. 1987. Elastin degradation in abdominal aortic aneurysms. Atherosclerosis. 65:13-21.

4. Newman, K.M., et al. 1994. Matrix metalloproteinases in abdominal aortic aneurysm: characterization, purification, and their possible sources. Connect. Tissue Res. 30:265-276.

5. Thompson, R.W., and Parks, W.C. 1996. Role of matrix metalloproteinases in abdominal aortic aneurysms. Ann. N. Y. Acad. Sci. 800:157-174.

6. Knox, J.B., Sukhova, G.K., Whittemore, A.D., and Libby, P. 1997. Evidence for altered balance between matrix metalloproteinases and their inhibitors in human aortic diseases. Circulation. 95:205-212.

7. Juvonen, J., et al. 1997. Elevated circulating levels of inflammatory cytokines in patients with abdominal aortic aneurysm. Arterioscler. Thromb. Vasc. Biol. 17:2843-2847.

8. Amento, E.P., Ehsani, N., Palmer, H., and Libby, P. 1991. Cytokines and growth factors positively and negatively regulate interstitial collagen gene expression in human vascular smooth muscle cells. Arterioscler. Thromb. 11:1223-1230.

9. Chandier, S., Cossins, J., Lury, J., and Wells, G. 1996. Macrophage metalloelastase degrades matrix and myelin proteins and processes a tumor necrosis factor-alpha fusion protein. Biochem. Biophys. Res. Commun. 228:421-429.

10. Schonbeck, U., Mach, F., and Libby, P. 1998. Generation of biologically active IL-1b by matrix metalloproteinases: a novel caspase-1-independent pathway of IL-1b processing. J. Immunol. 161:3340-3346.

11. Gilat, D., Cahalon, L., Hershkoviz, R., and Lider, O. 1996. Interplay of T cells and cytokines in the context of enzymatically modified extracellular matrix. Immunol. Today. 17:16-20.

12. Watanabe, T., Shimokama, T., Haraoka, S., and Kishikawa, H. 1995. T lymphocytes in atherosclerotic lesions. Ann. N. Y. Acad. Sci. 748:40-55.

13. Hansson, G.K., Holm, J., and Jonasson, L. 1989. Detection of activated Tlymphocytes in the human atherosclerotic plaque. Am. J. Pathol. 135:169-175.

14. Elser, B., et al. 2002. IFN-gamma represses IL-4 expression via IRF-1 and IRF-2. Immunity. 17:703-712.

15. Schonbeck, U., Sukhova, G.K., Gerdes, N., and
Libby, P. 2002. Th2 predominant immune responses prevail in human abdominal aortic aneurysm. Am. J. Pathol. 161:499-506.

16. Postlethwaite, A., Holness, M.A., Katai, H., and Raghow, R. 1992. Human fibroblasts synthesize elevated levels of extracellular matrix proteins in response to interleukin 4. J. Clin. Invest. 90:1479-1485.

17. Lacraz, S., et al. 1992. Suppression of metalloproteinase biosynthesis in human alveolar macrophages by interleukin-4. J. Clin. Invest. 90:382-388.

18. Shimizu, K., et al. 2001. Host bone-marrow cells are a source of donor intimal smooth- muscle-like cells in murine aortic transplant arteriopathy. Nat. Med. 7:738-741.

19. Shapiro, S.D., Endicott, S.K., Province, M.A., Pierce, J.A., and Campbell, E.J. 1991. Marked longevity of human lung parenchymal elastic fibers deduced from prevalence of D-aspartate and nuclear weaponsrelated radiocarbon. J. Clin. Invest. 87:1828-1834.

20. Thompson, R.W., et al. 1995. Production and localization of 92-kilodalton gelatinase in abdominal aortic aneurysms. An elastolytic metalloproteinase expressed by aneurysm-infiltrating macrophages. J. Clin. Invest. 96:318-326.

21. Vine, N., and Powell, J.T. 1991. Metalloproteinases 
in degenerative aortic disease. Clin. Sci. 81:233-239. 22. McMillan, W.D., et al. 1995. In situ localization and quantification of mRNA for $92-\mathrm{kD}$ type IV collagenase and its inhibitor in aneurysmal, occlusive, and normal aorta. Arterioscler. Thromb. Vasc. Biol. 15:1139-1144.

23. Pyo, R., et al. 2000. Targeted gene disruption of matrix metalloproteinase-9 (gelatinase B) suppresses development of experimental abdominal aortic aneurysms. J. Clin. Invest. 105:1641-1649.

24. Murphy, G., Cockett, M.I., Ward, R.V., and Docherty, A.J. 1991. Matrix metalloproteinase degradation of elastin, type IV collagen and proteoglycan. A quantitative comparison of the activities of $95 \mathrm{kDa}$ and $72 \mathrm{kDa}$ gelatinases, stromelysins- 1 and -2 and punctuated metalloproteinase (PUMP). Biochem. J. 277:277-279.

25. Shapiro, S.D., Kobayashi, D.K., Pentland, A.P., and Welgus, H.G. 1993. Induction of macrophage metalloproteinases by extracellular matrix. Evidence for enzyme- and substrate-specific responses involving prostaglandin-dependent mechanisms. J. Biol. Chem. 268:8170-8175.

26. Curci, J.A., Liao, S., Huffman, M.D., Shapiro, S.D., and Thompson, R.W. 1998. Expression and localization of macrophage elastase (matrix metalloproteinase-12) in abdominal aortic aneurysms. J. Clin. Invest. 102:1900-1910.

27. Punturieri, A., et al. 2000. Regulation of elastinolytic cysteine proteinase activity in normal and cathepsin K-deficient human macrophages. J. Exp. Med. 192:789-799.

28. Nagase, H. 1997. Activation mechanisms of matrix metalloproteinases. Biol. Chem. 378:151-160.

29. Freestone, T., et al. 1995. Inflammation and matrix metalloproteinases in the enlarging abdominal aortic aneurysm. Arterioscler. Thromb. Vasc. Biol. 15:1145-1151.

30. Libby, P., and Lee, R.T. 2000. Matrix matters. Circulation. 102:1874-1876.

31. Tilson, M.D. 1992. Aortic aneurysms and atherosclerosis. Circulation. 85:378-379.
32. Daugherty, A., Manning, M.W., and Cassis, L.A. 2000. Angiotensin II promotes atherosclerotic lesions and aneurysms in apolipoprotein E-deficient mice. J. Clin. Invest. 105:1605-1612.

33. Longo, G.M., et al. 2002. Matrix metalloproteinases 2 and 9 work in concert to produce aortic aneurysms. J. Clin. Invest. 110:625-632. doi:10.1172/ JCI200215334.

34. Shireman, P.K., et al. 1997. Elevations of tissue-type plasminogen activator and differential expression of urokinase-type plasminogen activator in diseased aorta. J. Vasc. Surg. 25:157-164.

35. Carmeliet, P., et al. 1997. Urokinase-generated plasmin activates matrix metalloproteinases during aneurysm formation. Nat. Genet. 17:439-444.

36. Huaux, F., Liu, T., McGarry, B., Ullenbruch, M., and Phan, S.H. 2003. Dual roles of IL-4 in lung injury and fibrosis. J. Immunol. 170:2083-2092.

37. Corcoran, M.L., Stetler-Stevenson, W.G., Brown, P.D., and Wahl, L.M. 1992. Interleukin 4 inhibition of prostaglandin E2 synthesis blocks interstitial collagenase and 92-kDa type IV collagenase/gelatinase production by human monocytes. J. Biol. Chem. 267:515-519.

38. Lanone, S., et al. 2002. Overlapping and enzyme-specific contributions of matrix metalloproteinases- 9 and -12 in IL-13-induced inflammation and remodeling. J. Clin. Invest. 110:463-474. doi:10.1172/ JCI200214136.

39. Furukawa, Y., et al. 1999. Interleukin-10 (IL-10) augments allograft arterial disease: paradoxical effects of IL-10 in vivo. Am. J. Pathol. 155:1929-1939.

40. Wang, Z., et al. 2000. Interferon gamma induction of pulmonary emphysema in the adult murine lung. J. Exp. Med. 192:1587-1600.

41. Shapiro, S.D., Campbell, E.J., Kobayashi, D.K., and Welgus, H.G. 1990. Immune modulation of metalloproteinase production in human macrophages. Selective pretranslational suppression of interstitial collagenase and stromelysin biosynthesis by interferon-gamma. J. Clin. Invest. 86:1204-1210.

42. Xie, B., et al. 1994. Localization of accessory path- ways from the 12-lead electrocardiogram using a new algorithm. Am. J. Cardiol. 74:161-165.

43. Kumar, R., Dong, Z., and Fidler, I.J. 1996. Differential regulation of metalloelastase activity in murine peritoneal macrophages by granulocytemacrophage colony-stimulating factor and macrophage colony-stimulating factor. J. Immunol. 157:5104-5111.

44. Gupta, S., et al. 1997. IFN-gamma potentiates atherosclerosis in ApoE knock-out mice. J. Clin. Invest. 99:2752-2761.

45. Nagano, H., et al. 1997. Interferon-gamma deficiency prevents coronary arteriosclerosis but not myocardial rejection in transplanted mouse hearts. J. Clin. Invest. 100:550-557.

46. Presti, R.M., Pollock, J.L., Dal Canto, A.J., O'Guin, A.K., and Virgin, H.W., 4th. 1998. Interferon gamma regulates acute and latent murine cytomegalovirus infection and chronic disease of the great vessels. J. Exp. Med. 188:577-588.

47. Weck, K.E., et al. 1997. Murine gamma-herpesvirus 68 causes severe large-vessel arteritis in mice lacking interferon-gamma responsiveness: a new model for virus-induced vascular disease. Nat. Med. 3:1346-1353.

48. Huang, S., et al. 1993. Immune response in mice that lack the interferon-gamma receptor. Science. 259:1742-1745.

49. Shichiri, M., and Hirata, Y. 2001. Antiangiogenesis signals by endostatin. FASEB J. 15:1044-1053.

50. Nakajima, K., Powers, J.C., Ashe, B.M., and Zimmerman, M. 1979. Mapping the extended substrate binding site of cathepsin $G$ and human leukocyte elastase. Studies with peptide substrates related to the alpha 1-protease inhibitor reactive site. J. Biol. Chem. 254:4027-4032.

51. Shimizu, K., Schonbeck, U., Mach, F., Libby, P., and Mitchell, R.N. 2000. Host CD40 ligand deficiency induces long-term allograft survival and donorspecific tolerance in mouse cardiac transplantation but does not prevent graft arteriosclerosis. J. Immunol. 165:3506-3518. 